\title{
On the Maximum Coverage Area of Wireless Networked Control Systems under Stability and Cost-Efficiency Constraints
}

\author{
Deniz Kilinc Mustafa Ozger Ozgur B. Akan \\ Next-generation and Wireless Communications Laboratory (NWCL) \\ Department of Electrical and Electronics Engineering \\ Koc University, Istanbul, Turkey \\ Email: \{dkilinc, mozger, akan\}@ku.edu.tr
}

\begin{abstract}
The integration of wireless communication and control systems revealed wireless networked control systems (WNCSs). One fundamental problem in WNCSs is to have a wide coverage area. For the first time in the literature, we address this problem and we obtain the maximum coverage area by solving an optimization problem. In this paper, we consider a WNCS where the output sensor measurements are transmitted over separate multi-hop wireless ad-hoc subnetworks. The system state is estimated using the Kalman filter. We present the critical arrival probability for a sensor measurement packet such that if the packet arrival probability is larger than the critical value, it is guaranteed that the expected state estimation error covariance is bounded, and hence the WNCS is stable. We find the optimum hop-diameter of a multi-hop wireless ad-hoc subnetwork under the constraints of both the stability of the WNCS and the cost-efficiency of the multi-hop wireless network. Furthermore, under these constraints, we derive the maximum total coverage area of the wireless subnetworks. The numerical analyses show that the maximum total coverage area can be increased by appropriately adjusting the number of sensors, the successful packet transmission probability between relay nodes, and the eigenvalues of the system matrix.
\end{abstract}

\section{INTRODUCTION}

Recent developments on micro sensor integrated systems have enabled combination of communication and control systems. This integration revealed networked control systems (NCSs) where the communication system enables the sensor observation delivery [1], [2]. The control system components such as sensors, actuators and plants with wireless communication capabilities constitute a wireless networked control system (WNCS). The observations of the sensors deployed over a wide area are fed to the WNCS through a wireless network. The WNCSs have a wide application area such as smart grid, automatic management and navigation systems [3].

For the WNCS applications requiring large coverage areas, e.g., space and terrestrial exploration, navigation systems, the maximum achievable area of the wireless network which ensures the stability of the WNCS is crucial. To the best of

This work was supported in part by the Turkish Scientific and Technical Research Council under grant \#110E249, by the Turkish National Academy of Sciences Distinguished Young Scientist Award Program (TUBA-GEBIP), and by IBM through IBM Faculty Award. our knowledge, no attempt has yet been made to find the maximum coverage area of the wireless network under the stability of the WNCS constraint. For the first time in the literature, we address this problem and obtain the solution by solving an optimization problem. Although in [4], [5], the authors investigate the maximum coverage area problem for wireless networks, they do not consider the stability of a WNCS which utilizes these wireless networks. In this paper, we find the maximum coverage area of a wireless network by considering both the stability of the WNCS and the cost efficiency of the wireless network.

In our scenario, wireless sensor nodes are employed to observe the system behavior. We consider that the sensor measurements are transmitted to the controller over a multihop wireless ad-hoc network. However, measurement packets may be lost due to the unreliable wireless channel characteristics caused by the noise, collision, and congestion. Since the WNCSs rely on the observations of the sensors to estimate the state of the system, any loss of the sensor measurements degrades the stability of the WNCS.

We use the Kalman filter for the state estimation of the system. The Kalman filtering is a well investigated technique in control theory [6], [7]. In the classical sense, the Kalman filter uses all the observation data provided by the sensors for the state estimation. However, for the WNCSs, the observations may be lost due to wireless channel conditions as stated above. In [6], the Kalman filter is studied when the observations are intermittent; nevertheless, the authors do not consider statistical convergence behavior. In [7], the authors investigate the state estimation process, in which the sensor measurements are received or lost completely in a stochastic manner, and they show that if the probability of arrival of an observation is above a threshold, the expectation of the state estimation error covariance is bounded. In [8], the authors consider two sensors, and the measurement of each sensor is independently received or lost by the Kalman filter.

We present the general case of the system presented in [8]. The observation process is divided into $N$ parts and each part is independently and randomly received or lost by the Kalman 
filter. Thus, we consider $N$ separate multi-hop wireless ad-hoc subnetworks for our scenario and each subnetwork includes a sensor node. Based on the derivations presented in [8], we derive the critical arrival probability for the measurement of each sensor such that if the arrival probability of a sensor measurement is larger than the critical value, it is guaranteed that the expectation of the state estimation covariance is bounded and the system is stable; otherwise it is not stable.

The packet arrival probability decreases as the number of hops during the packet transmission increases. The maximum hop number of the shortest paths between any two node pairs in the network is the hop-diameter of the network. We show that there exists a critical hop-diameter of a subnetwork such that if the hop-diameter of the subnetwork is less than the critical hop-diameter, the WNCS is stable. Another significant parameter for the WNCS is the cost-efficiency of the multi-hop wireless network. Based on the solution of an optimization problem, we find both the optimum hop-diameter and the maximum coverage area of the multi-hop wireless ad-hoc networks under the constraints of both the stability of the WNCS and cost-efficiency of the networks.

The paper is organized as follows. In Section II, we describe the Kalman filtering with partial observation losses. In Section III, we present the multi-hop wireless ad-hoc network model and investigate the connectivity of the network. In Section IV, we derive the maximum coverage area of the multi-hop wireless ad-hoc network under the constraints of the stability and cost-efficiency of the WNCS. In Section V, we present the numerical analysis. The paper is concluded in Section VI.

\section{Kalman Filtering with Partial Observation LOSSES}

In a WNCS, the Kalman filter gathers sensor measurements from distinct sensors and each sensor node encodes its own observation into a single packet. However, some of the packets might be lost during the wireless data transmission. In [8], the authors present a state estimation process with partial observation losses considering that the observation process is divided into two parts which are transmitted over different wireless channels by two different sensor nodes. In this section, we present a general state estimation process, i.e., the observation process is divided into $N$ parts, with partial observation losses using the Kalman filter. In other words, the Kalman filter uses the output observations of $N$ independent sensors.

We consider a general multiple-input multiple-output (MIMO) discrete time linear time-invariant system which is described by the following system equations

$$
\begin{aligned}
\mathbf{x}_{t+1} & =A \mathbf{x}_{t}+\mathbf{w}_{t}, \\
{\left[\begin{array}{c}
\mathbf{y}_{1, t} \\
\vdots \\
\mathbf{y}_{N, t}
\end{array}\right] } & =\left[\begin{array}{c}
C_{1} \\
\vdots \\
C_{N}
\end{array}\right] \mathbf{x}_{t}+\left[\begin{array}{c}
\mathbf{v}_{1, t} \\
\vdots \\
\mathbf{v}_{N, t}
\end{array}\right]
\end{aligned}
$$

where $\mathbf{x}_{t} \in \mathcal{R}^{n}$ is the system state vector, $\mathbf{w}_{t} \in \mathcal{R}^{n}$ is the system disturbance vector, $A \in \mathcal{R}^{n \times n}$ is the system matrix, $\mathbf{y}_{1, t} \in \mathcal{R}^{m_{1}}, \mathbf{y}_{2, t} \in \mathcal{R}^{m_{2}}, \ldots, \mathbf{y}_{N, t} \in \mathcal{R}^{m_{N}}$ are

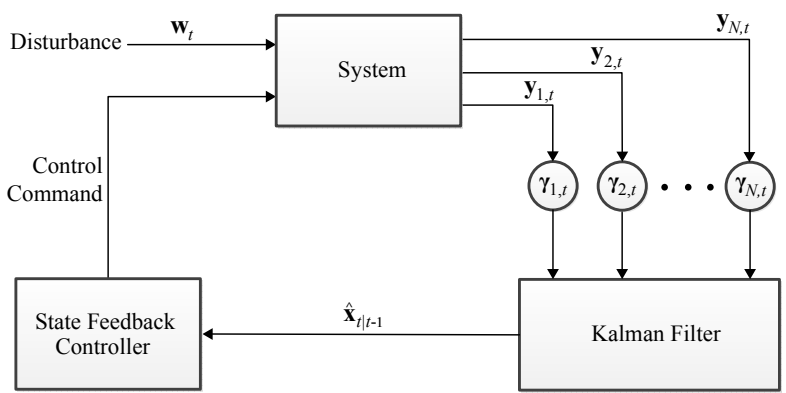

Fig. 1. The block diagram of the WCSN.

sensor measurement output vectors, $\mathbf{v}_{1, t} \in \mathcal{R}^{m_{1}}, \mathbf{v}_{2, t} \in \mathcal{R}^{m_{2}}$, $\ldots, \mathbf{v}_{N, t} \in \mathcal{R}^{m_{N}}$ are the measurement noise vectors, and $C_{1} \in \mathcal{R}^{m_{1} \times n}, C_{2} \in \mathcal{R}^{m_{2} \times n}, \ldots, C_{N} \in \mathcal{R}^{m_{N} \times n}$ are the output matrices. The subscript $t$ indicates the time index. Also note that the boldface symbols in this paper represent vectors. We define $\mathbf{y}_{t}=\left[\begin{array}{llll}\mathbf{y}_{1, t} ; & \mathbf{y}_{2, t} ; \ldots ; & \mathbf{y}_{N, t}\end{array}\right], \mathbf{v}_{t}=$ $\left[\mathbf{v}_{1, t} ; \mathbf{v}_{2, t} ; \ldots ; \mathbf{v}_{N, t}\right]$, and $C=\left[C_{1} ; C_{2} ; \ldots ; C_{N}\right]$. Both $\mathbf{w}_{t}$ and $\mathbf{v}_{t}$ are assumed to be Gaussian random vectors with zero mean and their covariance matrices are $Q \geq 0$ and $R>0$, respectively. $R$ is defined by $R=\left[\begin{array}{ccc}R_{11} & \ldots & R_{1 N} \\ \vdots & \ddots & \vdots \\ R_{N 1} & \ldots & R_{N N}\end{array}\right]$ where $R_{i j}=\mathrm{E}\left[\mathbf{v}_{i, t} \mathbf{v}_{j, t}^{\prime}\right]$. Furthermore, we assume that the system $(A, C)$ is observable; hence, the Kalman filter converges without sensor measurement losses.

The sensor measurement packets $\mathbf{y}_{1, t}, \mathbf{y}_{2, t}, \ldots, \mathbf{y}_{N, t}$ are encoded independently and transmitted over different multihop wireless ad-hoc subnetworks. We use random variable $\gamma_{i, t}$ which indicates whether the measurement packet of $i^{\text {th }}$ sensor, $\mathbf{y}_{i, t}$, is correctly received during a given sample period. We assume $\gamma_{i, t}$ for $i=1,2, \ldots, N$ are independent Bernoulli random variables with $\operatorname{Pr}\left\{\gamma_{i, t}=1\right\}=\lambda_{i}$ and $\operatorname{Pr}\left\{\gamma_{i, t}=0\right\}=1-\lambda_{i}$. That is, if $\gamma_{i, t}=1$, then the measurement packet $\mathbf{y}_{i, t}$ is correctly received; otherwise, the packet is lost during the wireless data transmission. The block diagram of the WNCS for our scenario is shown in Fig. 1. Note that the observation process is stochastic due to the random measurement losses during the packet transmission process.

Since we assume that $\gamma_{i, t}$ and $\gamma_{j, t^{\prime}}$ for $i \neq j$ are independent for every $t$ and $t^{\prime}$, the sensor measurement packets $\mathbf{y}_{i, t}$ for $i=$ $1,2, \ldots, N$ can be independently lost or received. Therefore, the loss of a measurement packet is equivalent to the reception of a measurement having an infinite noise variance. Then, for the measurement noise vectors $\mathbf{v}_{i, t}$, we define the following conditional probability distribution function

$$
f_{\mathbf{v} \mid \gamma}\left(\mathbf{v}_{i, t} \mid \gamma_{i, t}\right) \sim \begin{cases}\mathcal{N}\left(0, R_{i i}\right), & \text { if } \gamma_{i, t}=1 \\ \mathcal{N}\left(0, \sigma_{i}^{2} I\right), & \text { if } \gamma_{i, t}=0\end{cases}
$$

Then, we take the limit as $\sigma_{i}^{2} \rightarrow \infty$ to derive the Kalman filter equations in the case of random partial losses.

Let us define the vectors $\gamma_{t} \triangleq\left[\gamma_{1, t} ; \ldots ; \gamma_{N, t}\right], \gamma_{0}^{t} \triangleq$ 
$\left\{\gamma_{0}, \ldots, \gamma_{t}\right\}$, and $\mathbf{y}_{0}^{t}=\left\{\mathbf{y}_{0}, \ldots, \mathbf{y}_{N}\right\}$. Then, we define

$$
\begin{aligned}
\hat{\mathbf{x}}_{t \mid t} & \triangleq \mathrm{E}\left[\mathbf{x}_{t} \mid \mathbf{y}_{0}^{t}, \gamma_{0}^{t}\right], \\
P_{t \mid t} & \triangleq \mathrm{E}\left[\left(\mathbf{x}_{t}-\hat{\mathbf{x}}_{t \mid t}\right)\left(\mathbf{x}_{t}-\hat{\mathbf{x}}_{t \mid t}\right)^{\prime} \mid \mathbf{y}_{0}^{t}, \gamma_{0}^{t}\right], \\
\hat{\mathbf{x}}_{t+1 \mid t} & \triangleq \mathrm{E}\left[\mathbf{x}_{t+1} \mid \mathbf{y}_{0}^{t}, \gamma_{0}^{t}\right], \\
P_{t+1 \mid t} & \triangleq \mathrm{E}\left[\left(\mathbf{x}_{t+1}-\hat{\mathbf{x}}_{t+1 \mid t}\right)\left(\mathbf{x}_{t+1}-\hat{\mathbf{x}}_{t+1 \mid t}\right)^{\prime} \mid \mathbf{y}_{0}^{t}, \gamma_{0}^{t}\right] .
\end{aligned}
$$

For the Kalman filter, the time update and the observation processes are independent. Hence, for our scenario, the time update process of the Kalman filter is formulated based on the classical Kalman filter as $\hat{\mathbf{x}}_{t+1 \mid t}=A \hat{\mathbf{x}}_{t \mid t}$ and $P_{t+1 \mid t}=A P_{t \mid t} A^{\prime}+Q$. However, since the observation process is stochastic, the classical Kalman filter equations cannot be directly used for the measurement update process. Based on the results presented in [8], the state estimation error covariance $P_{t+1 \mid t}$ can be expressed in terms of $P_{t \mid t-1}$ as $P_{t+1 \mid t}=g\left(P_{t \mid t}\right)$ where $g(X)$ is defined in (4) at the bottom of this page, where $D_{i, j, \ldots, k}=\left[C_{i} ; C_{j} ; \ldots ; C_{k}\right]$, $F_{i, j, \ldots, k}=\operatorname{Cov}\left[\mathbf{v}_{i, t} ; \mathbf{v}_{j, t} ; \ldots ; \mathbf{v}_{k, t}\right]$. Because of the stochastic nature of the Kalman filter updates, a unique deterministic state estimation error covariance cannot be obtained in the steady state. Therefore, we consider the statistical properties of the state estimation error covariance of the Kalman filter.

In [7], the authors investigate the state estimation process, in which the sensor measurement packet is received or lost completely, and they show the existence of a critical packet arrival probability $\lambda^{c}$ such that $\mathrm{E}\left[P_{t+1 \mid t}\right]$ is bounded if $\lambda>\lambda^{c}$ and $\mathrm{E}\left[P_{t+1 \mid t}\right]$ becomes infinite as $t \rightarrow \infty$ if $\lambda<\lambda^{c}$. In addition, in [8], it is shown that for a state estimation process with random packet losses considering two measurement sensors, there is a critical packet arrival probability $\lambda_{1}^{c}$ of the measurement of the first sensor given the packet arrival probability $\lambda_{2}$ of the second sensor. For the general case, based on the derivations and results given in [8], if $(A, Q)$ is controllable and $(A, C)$ is observable, for a fixed set of $\left(\lambda_{1}, \lambda_{2}, \ldots, \lambda_{i-1}, \lambda_{i+1}, \ldots \lambda_{N}\right)$, if $\lambda_{i} \geq \lambda_{i}^{c}$, we can obtain positive semidefinite matrices $S \geq 0$ and $V \geq 0$ such that $0 \leq S \leq \lim _{t \rightarrow \infty} \mathrm{E}\left[P_{t+1 \mid t}\right] \leq V$, $\forall \mathrm{E}\left[P_{0}\right] \geq 0$ where $S=\left(1-\lambda_{1}\right) \ldots\left(1-\lambda_{N-1}\right) A S A^{\prime}+Q$ and $V=\mathrm{E}[g(V)]$. Therefore, the WNCS stable, if the state estimation error covariance is bounded. Furthermore, we know that $P_{t+1 \mid t}$ is bounded if and only if $\mathrm{E}\left[P_{t+1 \mid t}\right]$ is bounded. Thus, for a fixed set of $\left(\lambda_{1}, \lambda_{2}, \ldots, \lambda_{i-1}, \lambda_{i+1}, \ldots \lambda_{N}\right)$, the WNCS is stable if and only if $\lambda_{i} \geq \lambda_{i}^{c}$.

If the output matrices $C_{1}, C_{2}, \ldots, C_{N}$ are square and invertible $A$ has a single unstable eigenvalue, the upper and lower bounds for $\lim _{t \rightarrow \infty} \mathrm{E}\left[P_{t+1 \mid t}\right]$ coincide and the critical packet arrival probability of the measurement packet of the $i^{\text {th }}$ sensor becomes

$$
\lambda_{i}^{c}=\max \left\{0,1-\frac{1}{\alpha^{2}\left(\lambda_{1}, \lambda_{2}, \ldots, \lambda_{i-1}, \lambda_{i+1}, \ldots \lambda_{N}\right)}\right\}
$$

where $\alpha=\max _{i}\left|\sigma_{i}\right|$ and $\sigma_{i}$ is the $i^{\text {th }}$ eigenvalue of $A$ [8]. We discuss the appropriate selection of the set of $\left(\lambda_{1}, \lambda_{2}, \ldots, \lambda_{N}\right)$ in Section IV for a cost-efficient WNCS with the maximum coverage area under stability constraint.

\section{Multi-Hop Wireless Ad-Hoc Network Model AND CONNECTIVITY}

For the WNCS, we consider a multi-hop wireless ad-hoc network. The first advantage of multi-hop wireless ad-hoc networks is that they can be employed in a fast and easy way, which is the reason why they are named "ad-hoc networks" [9]. The second advantage of this network model is that very large areas can be covered by means of the multi-hop property. However, since the wireless channels are unreliable, as the number of hops increases during the packet transmission, the packet arrival probability decreases.

For our scenario, we assume that there are $N$ sensor nodes and each sensor transmits its measurement packet to the Kalman filter over a multi-hop wireless ad-hoc subnetwork. In addition, it is assumed that the nodes in each subnetwork are independently distributed according to a two dimensional Poisson point process. Each subnetwork is considered as separate from each other. In Fig. 2, the multi-hop wireless ad-hoc network model used in this paper is shown where $G_{i}$ denotes the $i^{\text {th }}$ subnetwork including the $i^{\text {th }}$ sensor node.

We consider that the transmission ranges of all sensor and relay nodes are the same and denoted by $r_{0}$. That is, if the distance between two nodes, $r$, satisfies $r \leq r_{0}$ condition, they are able to communicate directly via a wireless link. Furthermore, the successful packet transmission probability between two nodes, which are within the transmission range of each other, is assumed to be constant and the same for each transmission process in the network and it is denoted by $\beta$. Therefore, the probability that the $i^{\text {th }}$ sensor measurement is correctly received by the Kalman filter, i.e., $\lambda_{i}$, can be expressed as

$$
\lambda_{i}=\beta^{M_{i}}, \quad \text { for } i=1,2, \ldots, N
$$

where $M_{i}$ is the number of hops taken by the packet transmitted by the $i^{\text {th }}$ sensor until it reaches the Kalman filter.

$$
\begin{aligned}
g(X) & =A X A^{\prime}+Q \\
& -\gamma_{1, t} \gamma_{2, t} \ldots \gamma_{N, t} A X C^{\prime}\left(C X C^{\prime}+R\right)^{-1} C X A^{\prime} \\
& -\left(1-\gamma_{1, t}\right) \gamma_{2, t} \ldots \gamma_{N, t} A X D_{2, \ldots, N}^{\prime}\left(D_{2, \ldots, N} X D_{2, \ldots, N}^{\prime}+F_{2, \ldots, N}\right)^{-1} D_{2, \ldots, N} X A^{\prime} \\
& -\gamma_{1, t}\left(1-\gamma_{2, t}\right) \gamma_{3, t} \ldots \gamma_{N, t} A X D_{1,3, \ldots, N}^{\prime}\left(D_{1,3, \ldots, N} X D_{1,3, \ldots, N}^{\prime}+F_{1,3, \ldots, N}\right)^{-1} D_{1,3, \ldots, N} X A^{\prime} \\
& \ldots \\
& -\left(1-\gamma_{1, t}\right)\left(1-\gamma_{2, t}\right) \ldots\left(1-\gamma_{N-1, t}\right) \gamma_{N, t} A X D_{N}^{\prime}\left(D_{N} X D_{N}^{\prime}+F_{N}\right)^{-1} D_{N} X A^{\prime} .
\end{aligned}
$$




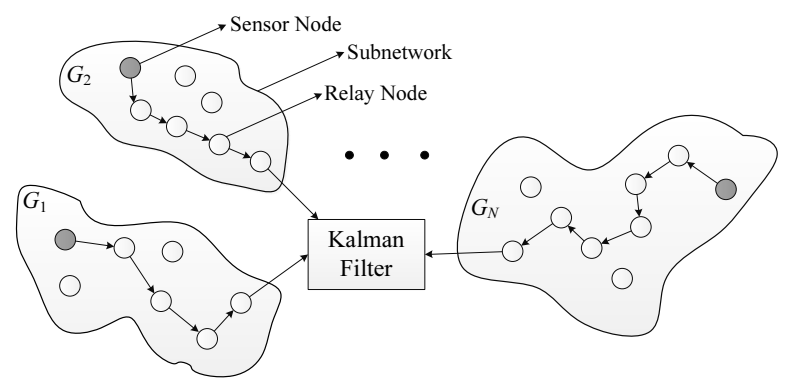

Fig. 2. The model of the multi-hop wireless ad-hoc subnetworks.

$M_{i}$ depends on the routing protocol, network topology, and number of nodes in the network.

A very fundamental and significant property of multi-hop wireless ad-hoc networks is the connectivity. To establish a fully connected ad-hoc network, a wireless multi-hop path from each node to each other node must exist. According to the results in [9], assuming that the nodes in the network are distributed according to a two dimensional Poisson point process, the probability that a multi-hop wireless ad-hoc network is $k$-connected is given by

$$
P_{k}=\left(1-\sum_{j=0}^{k-1} \frac{\left(\rho \pi r_{0}^{2}\right)^{j}}{j !} e^{-\rho \pi r_{0}^{2}}\right)^{m_{i}}
$$

for $i=1,2, \ldots, N$, where $P_{k}=\operatorname{Pr}\left\{G_{i}\right.$ is $k$-connected $\}, m_{i}$ is the total number of nodes in the subnetwork $G_{i}, \rho$ is the node density defined by $\rho=m / S_{i}$ where $S_{i}$ is the coverage area of $G_{i}$, and $r_{0}$ is the transmission range of a node. A network is said to be $k$-connected $(k=1,2,3, \ldots)$ if for each pair of nodes, there exist at least $k$ mutually independent links that connect them.

\section{Maximum Coverage Area Under Stability And COST-EFFICIENCY CONSTRAINTS}

In a multi-hop network, we can increase the coverage area by increasing the number of nodes in the network. However, if the coverage is enlarged with an increase in the number of nodes, the number of hops during the packet transmission between two distant nodes rises. Because of the unreliable wireless channels, an increase in the number of hops during the packet transmission decreases the packet arrival probability, and the WNCS might become unstable as discussed in Section II. Therefore, for a stable WNCS, the hop-diameter of the network becomes a critical parameter. In the paper, the hopdiameter of the subnetwork $G_{i}$ is denoted by $d_{i}$.

Let the critical packet arrival probability of the $i^{\text {th }}$ sensor measurement be $\lambda_{i}^{c}$. Based on the definition of the hopdiameter of a network, the maximum number of hop taken by a measurement packet until it reaches the Kalman filter is less than or equal to the hop-diameter of the subnetwork. Then, using (6), the critical-hop diameter of $i^{\text {th }}$ subnetwork is

$$
d_{i}^{c}=\left\lfloor\ln \left(\lambda_{i}^{c}\right) / \ln (\beta)\right\rfloor .
$$

That is, if the hop-diameter of the subnetwork $G_{i}$ satisfies $d_{i} \leq d_{i}^{c}$ condition, it is guaranteed that the arrival probability of the packet transmitted by $i^{\text {th }}$ sensor is larger than the critical arrival probability; hence, the WNCS is stable. However, if $d_{i}>d_{i}^{c}$, the stability of the system is not guaranteed. Since the hop-diameter depends on several factors such as topology, network size, node locations, sensor communication range, and node density, it is difficult to find a upper bound for the maximum number of nodes which ensures a given hopdiameter. Therefore, to guarantee the stability of the control system, one can use lower bound for the maximum number of nodes in a subnetwork given as $m_{i}\left(\lambda_{i}^{c}\right)=d_{i}^{c}+1$ where $m_{i}\left(\lambda_{i}^{c}\right)$ denotes the number of nodes which guarantees that the packet arrival probability is less than the critical value and the proof is straightforward.

If we consider only the stability criterion, for a given set of $\left(\lambda_{1}, \lambda_{2}, \ldots, \lambda_{i-1}, \lambda_{i+1}, \ldots \lambda_{N}\right)$, as $\lambda_{i}^{c} \rightarrow 0, m_{i}\left(\lambda_{i}^{c}\right) \rightarrow \infty$, and hence the total coverage area of the $i^{\text {th }}$ subnetwork becomes infinite. Indeed, it is irrational and cost-inefficient to place infinitely many nodes in a subnetwork including a sensor node whose critical packet arrival probability is 0 . That is, a decrease in $\lambda_{i}^{c}$ decreases the the importance of the subnetwork $G_{i}$, and when $\lambda_{i}^{c}=0$, the measurements of the $i^{\text {th }}$ sensor in $G_{i}$ become unnecessary for the WNCS. Thus, for the maximum coverage area of the multi-hop wireless network, it is not enough to consider only the stability of the WNCS. The costefficiency of the multi-hop wireless network should also be considered. In other words, a multi-hop wireless network for a stable WNCS might have infinite coverage area. However, such a multi-hop wireless network is cost-inefficient. As a result, the selection of a set of packet arrival probabilities of the sensor measurements, i.e., $\left(\lambda_{1}, \lambda_{2}, \ldots, \lambda_{N}\right)$, affects the cost-efficiency of the multi-hop wireless ad-hoc network.

Since when $\lambda_{i} \rightarrow 0, m_{i}\left(\lambda_{i}^{c}\right) \rightarrow \infty$ and $\lambda_{i} m_{i}\left(\lambda_{i}\right) \rightarrow 0$, we can use $\lambda_{i} m_{i}\left(\lambda_{i}\right)$ as the efficiency of the $i^{\text {th }}$ subnetwork. Therefore, to find a cost-efficient multi-hop wireless ad-hoc network, we define a cost-efficiency function as follows

$$
f\left(\lambda_{1}, \ldots, \lambda_{N}\right)=\lambda_{1} m_{1}\left(\lambda_{1}\right)+\cdots+\lambda_{N} m_{N}\left(\lambda_{N}\right)
$$

where $m_{i}\left(\lambda_{i}\right)$ is the number of nodes in the subnetwork $G_{i}$ which guarantees that the packet arrival probability is bounded above by $\lambda_{i}$ and it is given by $m_{i}\left(\lambda_{i}\right)=\left\lfloor\ln \left(\lambda_{i}\right) / \ln (\beta)\right\rfloor+1$. Note that the cost-efficiency function is the weighted sum of the number of nodes in the subnetworks. Using (5), the set of $\left(\lambda_{1}, \lambda_{2}, \ldots, \lambda_{N}\right)$ which both maximizes $f\left(\lambda_{1}, \ldots, \lambda_{N}\right)$ and ensures the stability of the WNCS can be found by solving the optimization problem in (10) at the top of the next page. The solution of (10) is given by

$$
\lambda_{i}^{\mathrm{opt}}=\max \left\{e^{-\ln (\beta)-1}, 1-\alpha^{-2 / N}\right\}
$$

for $i=1,2, \ldots, N$, where $\left(\lambda_{1}^{\mathrm{opt}}, \lambda_{2}^{\mathrm{opt}}, \ldots, \lambda_{N}^{\mathrm{opt}}\right)$ denotes the optimum stable set having the maximum cost-efficiency. The solution given in (11) satisfies the constraints of both the costefficiency and the stability. Then, using the optimum set of packet arrival probabilities given in (11), the optimum hop- 


$$
\begin{aligned}
& \operatorname{maximize}_{\lambda_{1}, \ldots, \lambda_{N}} f\left(\lambda_{1}, \ldots, \lambda_{N}\right)=\lambda_{1} m_{1}\left(\lambda_{1}\right)+\lambda_{2} m_{2}\left(\lambda_{2}\right)+\cdots+\lambda_{N} m_{N}\left(\lambda_{N}\right) \\
& \text { subject to } \lambda_{i}<\max \left\{0,1-\frac{1}{\alpha^{2}\left(1-\lambda_{1}\right) \ldots\left(1-\lambda_{i-1}\right)\left(1-\lambda_{i+1}\right) \ldots\left(1-\lambda_{N-1}\right)}\right\} \quad \text { for } i=1,2, \ldots, N
\end{aligned}
$$

diameter of the $i^{\text {th }}$ subnetwork having the maximum costefficiency is given by

$$
d_{i}^{\mathrm{opt}}=\left\lfloor\frac{\ln \left(\max \left\{e^{-\ln (\beta)-1}, 1-\alpha^{-2 / N}\right\}\right)}{\ln (\beta)}\right\rfloor .
$$

Furthermore, to guarantee the stability of the WNCS, we use the lower bound for the maximum number of nodes in $G_{i}$, denoted by $m_{i}\left(\lambda_{i}^{\mathrm{opt}}\right)$, and it is

$$
m_{i}\left(\lambda_{i}^{\mathrm{opt}}\right)=\left\lfloor\frac{\ln \left(\max \left\{e^{-\ln (\beta)-1}, 1-\alpha^{-2 / N}\right\}\right)}{\ln (\beta)}\right\rfloor+1
$$

for $i=1,2, \ldots, N$.

Now, we derive the number of nodes in each subnetwork under the stability and cost-efficiency constraints. To find the maximum coverage area of the subnetworks for the number of nodes given in (13), we consider the connectivity of the subnetworks. In (7), the probability that a multi-hop wireless ad-hoc network is $k$-connected is given. For a fixed number of nodes, we can say that 1 -connected network has the maximum coverage area from (7). Thus, to have the maximum coverage area for a given number of nodes, we set $k=1$. Then, using (7), for a stable WNCS, the maximum coverage area of the subnetwork $G_{i}$, which is 1 -connected and cost-efficient, is given by $S_{i}=\left[-m_{i}\left(\lambda_{i}^{\mathrm{opt}}\right) \pi r_{0}^{2}\right] /\left[\ln \left(1-P_{1}^{1 / m_{i}\left(\lambda_{i}^{\mathrm{opt}}\right)}\right)\right]$ where $P_{1}$ is the probability that $G_{i}$ is 1 -connected. Since the number of nodes found in (13) is the same for each subnetwork, the maximum total coverage area of the subnetworks is

$$
S^{\mathrm{T}}=\frac{-N m_{i}\left(\lambda_{i}^{\mathrm{opt}}\right) \pi r_{0}^{2}}{\ln \left(1-P_{1}^{1 / m_{i}\left(\lambda_{i}^{\mathrm{opt}}\right)}\right)} .
$$

Note that from (7), for a given number of nodes, to make the connectivity probability equal to 1 , the node density of the network must be infinite which is not realistic. However, if we set the probability that $G_{i}$ is 1 -connected as $P_{1}=0.99$, it can be said that the $G_{i}$ subnetwork is almost surely connected.

\section{Numerical Analysis}

In this section, we present the numerical analysis of both the optimum hop-diameter $d_{i}^{\text {opt }}$ of a subnetwork and the maximum total coverage area $S^{\mathrm{T}}$ of the subnetworks with respect to several parameters of the system and multi-hop wireless subnetworks. For the numerical analysis, we consider that the subnetwork $G_{i}$ is 1 -connected with probability $P_{1}=0.99$. Moreover, the output matrices $C_{1}, C_{2}, \ldots, C_{N}$ are assumed to be square and invertible.
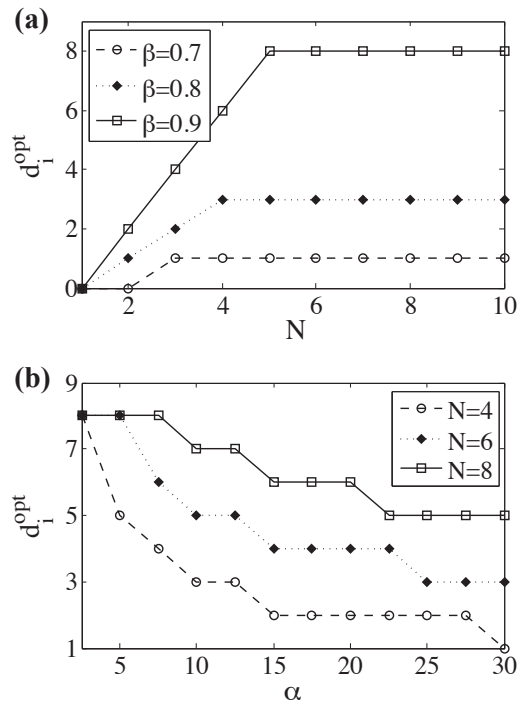

Fig. 3. $d_{i}^{\text {opt }}$ (a) with respect to $N$ for different $\beta$ values and (b) with respect to $\alpha$ for different $N$ values.

\section{A. Optimum Hop-Diameter}

In this part of the numerical analysis, we present the variation of the optimum hop-diameter, $d_{i}^{\text {opt }}$, given in (12) with respect to the number sensor nodes, $N$, the successful packet transmission probability between two nodes, $\beta$, and the eigenvalue of $A$ having the maximum magnitude, $\alpha$. For this part, we set the transmission range of the sensor and relay nodes in the multi-hop wireless subnetworks as $r_{0}=50 \mathrm{~m}$.

In Fig. 3(a), $d_{i}^{\text {opt }}$ with respect to the number of sensor nodes $N$ employed for the WNCS with different $\beta$ values is shown. $d_{i}^{\text {opt }}$ increases with an increase in $\beta$ which is an expected result. Note that $0 \leq \beta \leq 1$ and as $\beta \rightarrow 1$, $\ln (\beta) \rightarrow-\infty$, also the numerator in (12) is negative; hence, an increase in $\beta$ causes an increase in $d_{i}^{\text {opt }}$. As seen in Fig. 3(a), $d_{i}^{\text {opt }}$ increases up to $N=5$, then it becomes constant. If $N>-2 \ln (\alpha) / \ln \left(1-e^{-\ln (\beta)-1}\right)$, then $\max \left\{e^{-\ln (\beta)-1}, 1-\right.$ $\left.\alpha^{-2 / N}\right\}=e^{-\ln (\beta)-1}$, and hence $d_{i}^{\text {opt }}$ depends only on $\beta$. On the other hand, if $N<-2 \ln (\alpha) / \ln \left(1-e^{-\ln (\beta)-1}\right)$, then $\max \left\{e^{-\ln (\beta)-1}, 1-\alpha^{-2 / N}\right\}=1-\alpha^{-2 / N}$; thus, $d_{i}^{\text {opt }}$ depends on $\alpha$ and $N$, i.e., $d_{i}^{\text {opt }}=\ln \left(1-\alpha^{-2 / N}\right) / \ln (\beta)$. Obviously, $d_{i}^{\text {opt }}$ decreases with an increase in $\alpha$. For a fixed $\beta=0.9$, the results seen in Fig. 3(b) show that $d_{i}^{\text {opt }}$ decreases with an increase in $\alpha$, which supports our inferences. It is also seen that $d_{i}^{\mathrm{opt}}$ can be increased with an increase in $N$.

\section{B. Maximum Total Coverage Area}

In this section of the numerical analysis, we present the effect of $N, \beta, \alpha$ on the maximum total coverage area of the 
(a)

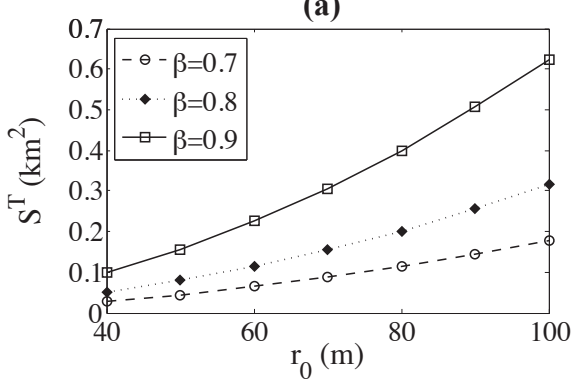

(b)

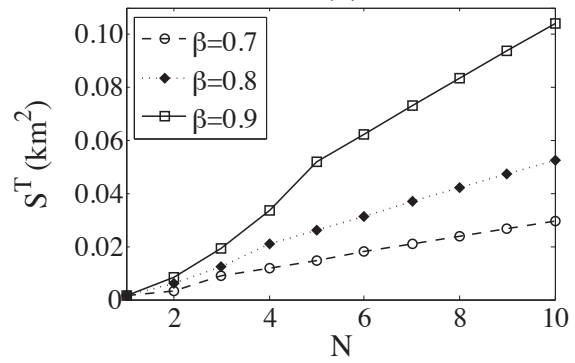

(c)

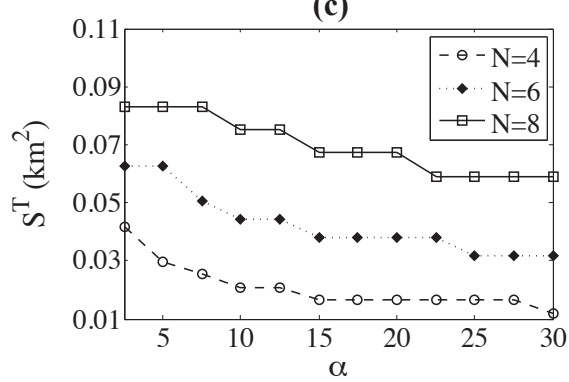

Fig. 4. $S^{\mathrm{T}}$ with respect to (a) $r_{0}$ for different $\beta$ values, (b) $N$ for different $\beta$ values, and (c) $\alpha$ for different $N$ values.

subnetworks, $S^{\mathrm{T}}$, given in (14). In Fig. 4(a), for constant $N=$ 15 and $\alpha=4.0$, the variation of the maximum total coverage area $S^{\mathrm{T}}$ with respect to $r_{0}$ is illustrated for different $\beta$ values. $S^{\mathrm{T}}$ is proportional with $r_{0}^{2}$ as seen in (14) and the quadratic dependence on $r_{0}$ can be seen in Fig. 4(a). In addition, the results show that an increase in $\beta$ enlarges the total coverage area of the subnetworks. From Section V-A, we know that $d_{i}^{\text {opt }}$ increases with an increase in $\beta$, and $m_{i}\left(\lambda_{i}^{\text {opt }}\right)=d_{i}^{\text {opt }}+1$. Thus, from (14), it is obvious that $S^{\mathrm{T}}$ becomes larger with an increase in $d_{i}^{\text {opt }}$.

In Fig. 4(b), for fixed $r_{0}=50 \mathrm{~m}$ and $\alpha=4.0$, the variation of $S^{\mathrm{T}}$ with respect to the number of sensor nodes used for the WNCS for varying $\beta$ values is demonstrated. According to the results, an increase in $\beta$ enlarges the total coverage area of the subnetworks because of the same reasons discussed above. Furthermore, $S^{\mathrm{T}}$ becomes larger with an increase in the number of sensors $N$ as shown in Fig. 4(b), which is an expected result because $S^{\mathrm{T}}=N S_{i}$. Note also that, for each $\beta$ value, $S^{\mathrm{T}}$ increases in a quadratic trend up to $N=$ 5 ; afterwards, it increases linearly with $N$. In Fig. 3(a), it is shown that up to $N=5, d_{i}^{\text {opt }}$ rises with an increase in $N$, which causes an increase in the maximum coverage area of a single subnetwork $S_{i}$ and we know that $S^{\mathrm{T}}=N S_{i}$. As a result, up to $N=5, S^{\mathrm{T}}$ increases quadratically. For $N>5$, since $d_{i}^{\text {opt }}$ becomes constant, $S_{i}$ also becomes constant. Thus, for $N>5, S^{\mathrm{T}}$ increases linearly with $N$ as seen in Fig. 4(b).

The effect of $\alpha$, i.e., the eigenvalue of the system matrix $A$ having the maximum magnitude, on the maximum total coverage area of the multi-hop wireless subnetworks $S^{\mathrm{T}}$ is shown in Fig. 4(c) for different $N$ values. Here, we set $r_{0}=$ $50 \mathrm{~m}$ and $\beta=0.9$. According to the results, an increase in $\alpha$, causes a reduction in the maximum total coverage area $S^{\mathrm{T}}$. As we state previously, if $N>-2 \ln (\alpha) / \ln \left(1-e^{-\ln (\beta)-1}\right)$, then $d_{i}^{\text {opt }}=\ln \left(1-\alpha^{-2 / N}\right) / \ln (\beta)$. That is, for a given $N$, an increase in $\alpha$ decreases the optimum hop-diameter. Therefore, since $m_{i}\left(\lambda_{i}^{\mathrm{opt}}\right)=d_{i}^{\mathrm{opt}}+1$, an increase in $\alpha$ also decreases $S^{\mathrm{T}}$, which can be seen in (14). Moreover, as illustrated in the figure, $S^{\mathrm{T}}$ increases with an increase in $N$, which is discussed in detail above.

\section{CONCLUSION}

In this paper, we investigate the multi-hop wireless ad-hoc network for a WNCS with multiple sensors. We present the critical arrival probability for the measurement packet of a sensor such that if the probability of arrival of the packet is larger than the critical value, the state estimation error covariance is bounded and the system is stable. We derive the expressions for both the optimum hop-diameter and the maximum coverage area of the multi-hop wireless subnetworks under the constraints of the stability and cost-efficiency. For the WNCS applications requiring wide coverage areas, e.g., space and terrestrial exploration and navigation systems, the maximum coverage area expression can be used to construct a cost-efficient multi-hop network which ensures the stability of the control system.

The numerical analyses show that both the optimum hopdiameter and the total coverage area of the subnetworks can be increased with an increase in the successful packet transmission probability between two nodes. Furthermore, for the WNCS, increasing the number of sensors rises the optimum hop-diameter and the maximum coverage area. According to the results, an decrease in the eigenvalue of the system matrix with maximum magnitude increases both the optimum hopdiameter and the total coverage area of the subnetworks.

\section{REFERENCES}

[1] Y. Halevi and A. Ray, "Integrated communication and control systems: Part I-Analysis” J. Dynamic Syst., Measure. Contr., vol. 110, pp. 367-373, Dec. 1988.

[2] M.S. Branicky, S.M. Phillips, and W. Zhang, "Stability of networked control systems: Explicit analysis of delay", in Proc. Amer. Control Conf., Chicago, IL, June 2000, pp. 2352-2357.

[3] A. Bemporad, M. Johansson, and M. Heemels, Networked Control Systems. Berlin, Germany: Springer, 2010.

[4] C. F. Huang and Y. C. Tseng, "The coverage problem in a wireless sensor network," in Proc. ACM Int. Conf. Wireless Sensor Networks and Applications (WSNA), 2003, pp. 115-121.

[5] C. C. Tseng and K. C. Chen, "Power Efficient Topology Control in Wireless Ad Hoc Networks," in Proc. IEEE Wireless Comm. and Networking Conf. (WCNC), 2004, pp. 610-615.

[6] T. Fortmann, Y. Bar-Shalom, M. Scheffe, and S. Gelfand, "Detection thresholds for tracking in cluttera connection between estimation and signal processing," IEEE Trans. Automat. Contr., vol. AC-30, pp. 221 228, Mar. 1985.

[7] B. Sinopoli, L. Schenato, M. Franceschetti,K. Poola, M. I. Jordan, and S. S. Sastry, "Kalman filtering with intermittent observations", IEEE Trans. Autom. Control, vol. 49, no. 9, pp. 1453-1464, Sep. 2004.

[8] X. Liu and A. Goldsmith, "Kalman filtering with partial observation losses", in Proc. IEEE Conf. Decision and Control, Bahamas, Dec. 2004, vol. 4, pp. 4180-4186.

[9] C. Bettstetter, "On the Minimum Node Degree and Connectivity of A Wireless Multihop Network," in Proc. ACM Intl Symp. Mobile Ad Hoc Networking and Computing, Switzerland, June 2002, pp. 80-91. 